\title{
RADAR REMOTE SENSING APPLICATIONS IN LANDSLIDE MONITORING WITH MULTI-PLATFORM INSAR OBSERVATIONS: A CASE STUDY FROM CHINA
}

\author{
T. Qu ${ }^{1,2}, \mathrm{Q} . \mathrm{Xu}^{2}, \mathrm{C} \cdot \mathrm{Liu}^{3, *}, \mathrm{Z} \cdot \mathrm{Li}^{4}$, B. Chen ${ }^{1}, \mathrm{~K} . \mathrm{Dai}^{2}$ \\ ${ }^{1}$ College of Engineering, Peking University, 100871 Beijing, China - (tengteng.qu, pkuchenbo, yangmk)@pku.edu.cn \\ ${ }^{2}$ State Key Laboratory of Geohazard Prevention and Geoenvironment Protection, Chengdu University of Technology, Chengdu \\ 610059, China-(xq, daikeren17)@cdut.edu.cn \\ ${ }^{3}$ College of Surveying and Geo-Informatics, Tongji University, Shanghai 200092, China - liuchun@tongji.edu.cn \\ ${ }^{4}$ COMET, School of Engineering, Newcastle University, Newcastle Upon Tyne NE1 7RU, UK - zhenhong.li@newcastle.ac.uk
}

\section{Commission III, WG III/3}

KEY WORDS: Radar Remote Sensing, InSAR, Multi-platform, TerraSAR-X, Sentinel-1, Landslide Monitoring

\begin{abstract}
:
In China, landslides are widely distributed in the mountainous areas of western regions. Especially after the Wenchuan Earthquake in 2008, a large number of landslides were triggered. This work focuses on the deformation monitoring of Xishancun Landslide based on multi-platform spaceborne radar remote sensing techniques. The spatio-temporal deformation characteristics of landslide could be retrieved from time series InSAR processing with joint use of Sentinel-1 and TerraSAR-X datasets. Eventually, the deformation and evolution histories are cultivated thoroughly to realize an effective and comprehensive monitoring and research of Xishancun Landslide. This work concludes that spaceborne radar remote sensing applications could demonstrate great potentials to identify the spatio-temporal characteristics and investigate the kinematics for hazardous landslides, especially combined with in situ measurements and other remote sensing observations.
\end{abstract}

\section{INTRODUCTION}

Landslide is one of the major and most frequently occurring geo-hazards around the world. After the 2008 Wenchuan Earthquake in China, a series of large-scale landslides were triggered (Yin, 2009). Unexpectedness and concealed nature of the landslides significantly increase the destruction degree and difficulty to prevent, exposing people's livelihoods and infrastructure at risk (Huang et al., 2009; Liu et al., 2013).

The acquisition and long-term monitoring of surface deformation information of large-scale landslides is an important basis for early warning and prediction of landslides (Xu et al., 2008). Especially, spaceborne radar remote sensing could realize macro dynamic monitoring of large-scale landslide hazards and provide an efficient way to obtain landslide surface deformation and spatio-temporal characteristics, hence contribute to early detection and early warning for local disaster risk management (Ye et al., 2004; Zhao et al., 2012).

As an important part of ESA Copernicus program, ESA launched Sentinel-1A on April 3, 2014 and Sentinel-1B on April 16, 2016, opening a new era of radar satellite observations. Sentinel-1 has a maximum resolution of 5 meters and a single-satellite re-visit cycle of 12 days, with Sentinel-1B and Sentinel-1A in the same orbit (Torres et al., 2012). Because of the precise orbital cube of Sentinel-1, the spatial baselines could be always kept within a certain range, which greatly improves the coherence of radar image acquisitions, and provides an important support for landslide monitoring and emergency response. Since its launch, many research achievements have been demonstrated in landslide monitoring. In 2016, Dai et al. used Sentinel-1 images to monitoring the activities of Daguangbao mega-landslide using time series interferometry (Dai et al., 2016). In 2017, Barra et al. used Sentinel-1 images and differential interferometry technique to map and monitor the deformation activity in La Gomera, Tenerife and Gran Cananria islands (Barra et al. 2017). Intrieri et al. used Sentinel-1 images for time series InSAR processing to obtain the pre-disaster deformation of Maoxian Landslide in Sichuan, China (Intrieri et al., 2018). Fiaschi et al. used Sentinel-1A datasets to detect and monitor landslides in the Veneto region of Italy (Fiaschi et al., 2017). Czikhardt et al. used Sentinel-1 images and time series InSAR technique to monitor ground stability in landslide-prone areas of Slovenia (Czikhardt et al., 2017). In 2018, Kyriou et al. assessed the applicability of Sentinel-1 data in landslide mapping using three different processing methods, that is, interferogram map generation, DSM comparisons before and after landslide, and offset tracking analysis (Kyriou and Nikolakopoulos, 2018). Liu et al. deployed Sentienl-1 datasets as well as other radar satellite observations for mutli-temporal loess landslide inventory mapping (Liu et al., 2018).

This work shares radar remote sensing applications in landslide monitoring case study in Sichuan Province of China since 2014. Long deformation evolutions of landslide area were retrieved from time series InSAR processing with joint use of multi-platform InSAR observations using TerraSAR-X StripMap datasets and Sentinel-1A/B TOPS datasets.

* Corresponding author 


\section{GEOLOGICAL SETTING OF XISHANCUN LANDSLIDE}

The Xishancun Landslide (Figure 1) is located in Li County, Sichuan Province, southwest of China and only $20 \mathrm{~km}$ away from Wenchuan County. This large-scale landslide is seriously affected by the "5.12" Wenchuan Earthquake in 2008. Its rear edge is bounded by near-vertical cliffs, and the front edge of the landslide is bounded by Zagunaohe River. There are gullies on both sides of the landslide. The plane shape of the landslide body is generally circular chair-like, with the leading edge elevation of $1510 \mathrm{~m}$, the trailing edge elevation of $3300 \mathrm{~m}$ and the height difference of $1790 \mathrm{~m}$. The length of landslide is about $3800 \mathrm{~m}$, the minimum width is $680 \mathrm{~m}$ and the maximum is $980 \mathrm{~m}$. The average thickness of the sliding body is $55 \mathrm{~m}$, and the scale of the sliding body is $1.7 \times 10^{8} \mathrm{~m}^{3}$.

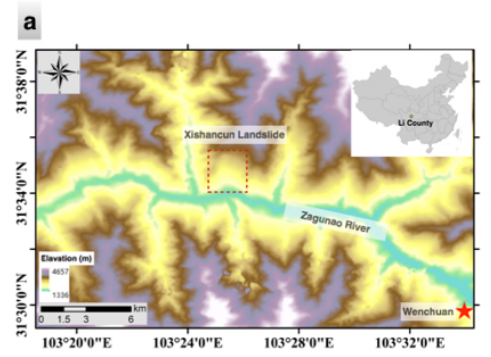

b

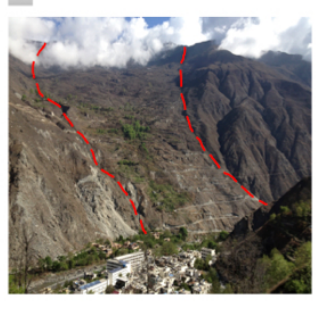

Figure 1. The overview of Xishancun Landslide

The whole vegetation in the landslide area is scarce, with a typical arid landscape.The middle and lower parts of the landslide are rich in water catchment and domestic water, groundwater is relatively rich, vegetation is developed, fruit trees are numerous, and crops are flourishing. Due to the construction of mountain-village highway, the cutting slope at the foot of the front slope of the landslide is serious, which has formed a steep slope of $3-15 \mathrm{~m}$, and part of the area has collapsed. The complexity of topographic conditions and slope gradient are the main factors controlling the occurrence of geological hazards, which are further deteriorated under the damage of the "5.12" Wenchuan earthquake. A Spatial Sensor Network (Figure 2) is also deployed on Xishancun Landslide with multiple boreholes and in-situ sensors since 2014 (Qu et al., 2016; Liu et al., 2018).

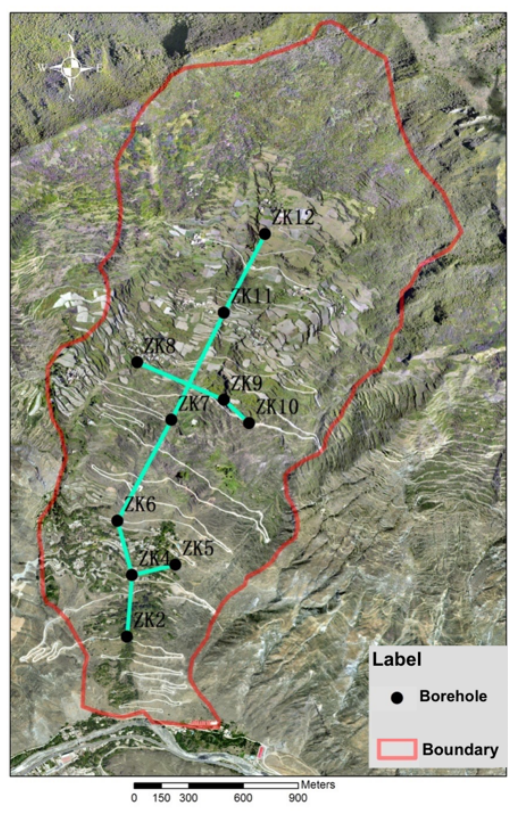

Figure 2. Spatial distributions of diverse boreholes on Xishancun Landslide

\section{MULTI-PLATFORM TIME SERIES INSAR PROCESSING}

In this study, TerraSAR-X StripMap descending datasets acquired from Oct. 2014 to April. 2015 and Sentinel-1A/B TOPS descending datasets acquired from Oct. 2014 to Feb. 2017 are utilized for Time Series InSAR processing. As mountainous western China has undulating terrains and abundant water vapor on valley slopes, which resulted in the severe problems of atmospheric disturbance in time series InSAR processing, TSInSAR-AEM method (Li et al., 2009) proposed by Zhenhong $\mathrm{Li}$ is deployed with integration of atmospheric phase estimation, which accurately separated the atmospheric disturbance and improved the reliability of time series deformation results.

Sentinel-1 uses the most advanced precise orbit determination technology, and its orbit accuracy is more precise than any previous radar satellite. It can be seen from Figure 3 that the perpendicular baselines of Sentinel-1 interferometric pairs are significantly shorter than those of TerraSAR-X pairs. The longest Sentinel-1 baseline is 212 meters, while the interferometric pairs with baselines under 100 meters account for $80 \%$. Short baseline greatly guarantees the coherence quality of interferometric pairs, and provides a reliable data basis for subsequent differential processing.
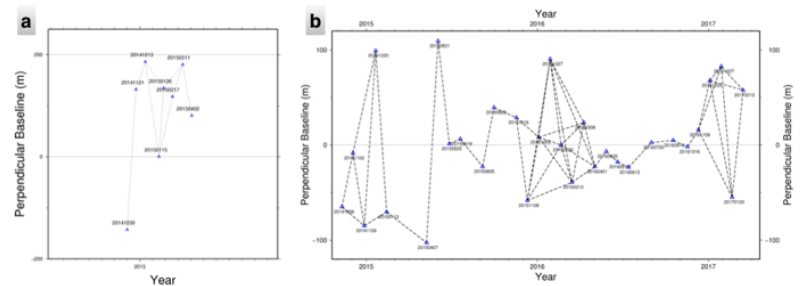

Figure 3. Spatial (vertical axis) and temporal (horizontal axis) baselines of TerraSAR-X (a) and Sentinel-1 (b) datasets during the TSInSAR-AEM processing 
The displacement rate maps computed using TerraSAR-X datasets is demonstrated in image a of Figure 4. The middle part of the landslide accounted for largest deformation with a rate of $13.2 \mathrm{~cm} / \mathrm{yr}$. The eastern boundary of the deformation slope is quite clear with the displacement rate being $10 \mathrm{~cm} / \mathrm{yr}$. The upper part of the slope is relatively stable, with no obvious deformation signals. The lower part of the slope deformed significantly with a rate of $8 \mathrm{~cm} / \mathrm{yr}$. A small region quite close to the river bank demonstrated a displacement rate of $11 \mathrm{~cm} / \mathrm{yr}$.

The displacement rate map computed using Sentinel-1 descending datasets is demonstrated in image $\mathrm{b}$ of Figure 4. As a whole, the deformation patterns of Xishancun landslide using Sentinel-1 datasets are quite similar to the patterns acquired from TerraSAR-X processing. However, the overall displacement rate is relatively lower than the result shown above. The largest deformation area is still the middle part of the slope, with a rate of $12.4 \mathrm{~cm} / \mathrm{yr}$. The eastern boundary of the deformation slope shows a displacement rate being $8 \mathrm{~cm} / \mathrm{yr}$. The lower part of the landslide demonstrated a creeping displacement rate of only $4 \mathrm{~cm} / \mathrm{yr}$. a)

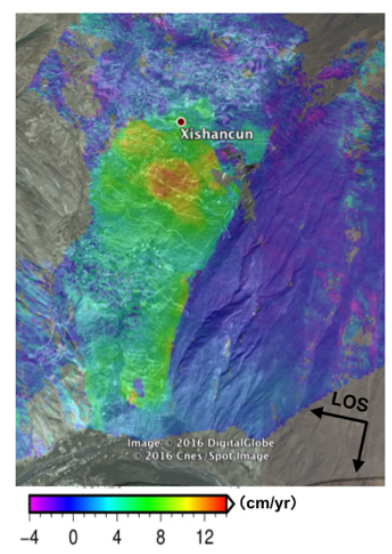

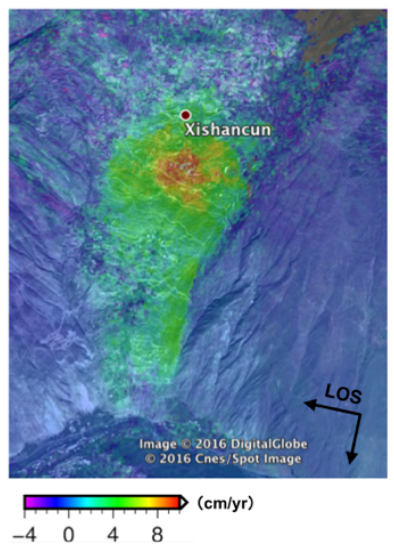

Figure 4. Deformation displacement rate maps derived from TerraSAR-X (a) and Sentinel-1 (b) datasets

Disparities between TerraSAR-X and Sentinel-1 time series results could be caused by: 1) Limited time span for TerraSAR-X results in its overestimate of annual velocity. 2) Different temporal samplings. 3) Different observation geometries. 4) DEM-induced uncertainties.

Daily rainfall data from 2014 to 2016 can be obtained due to the installation of rainfall gauges on landslides. In order to evaluate the reliability of the atmospheric phase estimation results, it is necessary to analyze the influence of the atmospheric phase screen on the deformation monitoring results of Xishancun Landslide. As Xishancun Landslide is near Zagunao River and located in the mountainous area with large relief, the phase delay caused by the atmospheric phase screen is very significant. Figure 5 shows the cumulative atmospheric phase correction of ZK2, the borehole closest to Zagunao River in the front of landslide body. It can be seen from the chart that the time series atmospheric phase corrections of ZK2 and daily rainfall produce almost synchronous trough peaks during the flood seasons in 2015 and 2016, hence it can be inferred that the atmospheric phase values and rainfall have a high correlation.

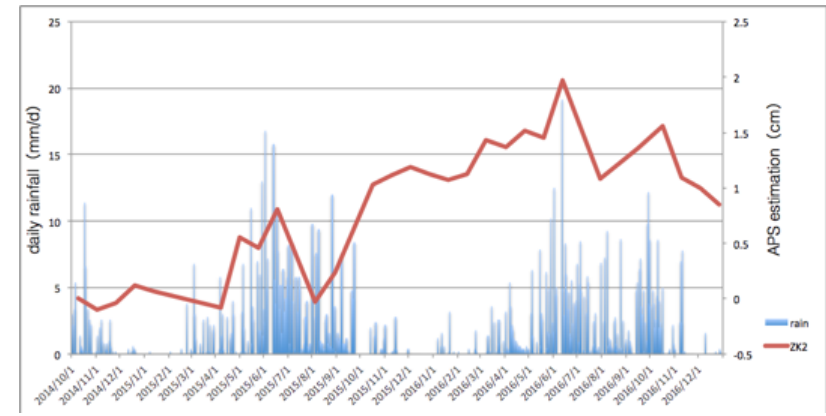

Figure 5. The relationship of rainfall and accumulative atmospheric phase corrections achieved by time series processing on Borehole ZK2

The radar images acquired during the rainy season will cause serious atmospheric artifacts, while the contribution of the atmospheric phase screen for the radar images acquired during the dry seasons is relatively small. Therefore, the application of space-borne radar remote sensing in landslide deformation monitoring needs to focus on the estimation and removal of atmospheric artifacts. The TSInSAR-AEM model used in this paper effectively avoids introducing atmospheric artefacts into time series deformation signals, thus facilitating the interpretation and analysis of the final deformation evolution results.

\section{TIME SERIES EVOLUTIONS OF XISHANCUN LANDSLIDE ACQUIRED FROM MULTI-PLATFORM OBSERVATIONS}

Figure 6 and Figure 7 shows the time series deformation evolutions of Xishancun landslide using TerraSAR-X and Sentinel-1 datasets since 2014. Especially, as Sentinel-1 datasets cover a long time span, the evolution of Xishancun Landslide has been fully demonstrated.
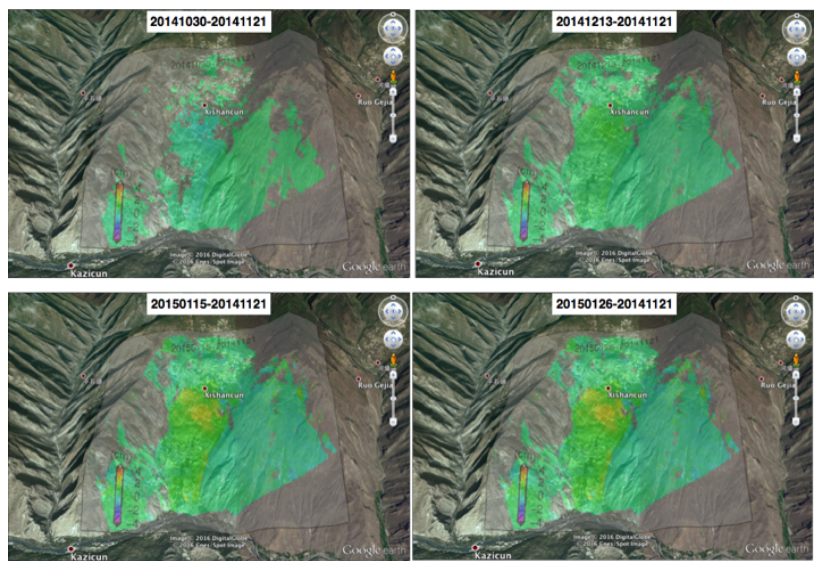


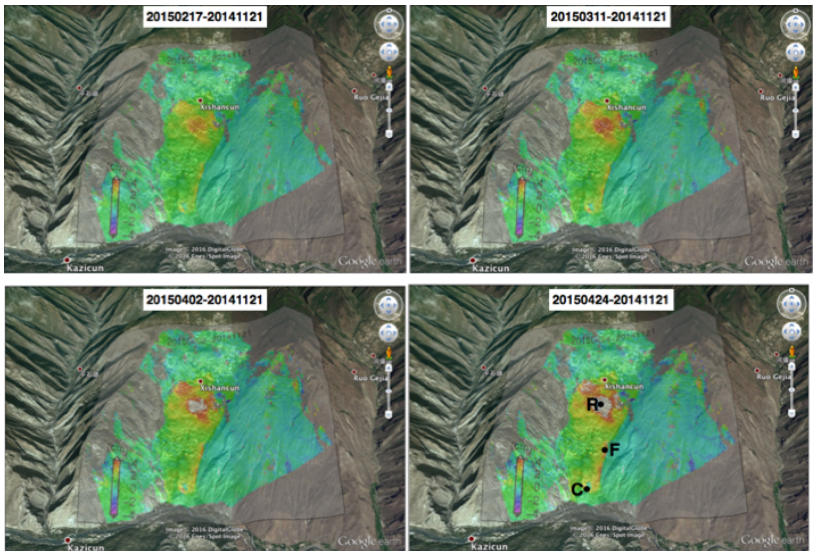

Figure 6. Time series evolutions of Xishancun Landslide acquired from TerraSAR-X datasets

From the time series results from multi-platform radar observations, it can be concluded that the middle and eastern parts of the landslide body deformed for two and a half years, but the overall deformation range did not expand significantly. The front edge and rear edge of the landslide body did not have obvious deformations, and the whole landslide was still in the creeping stage.

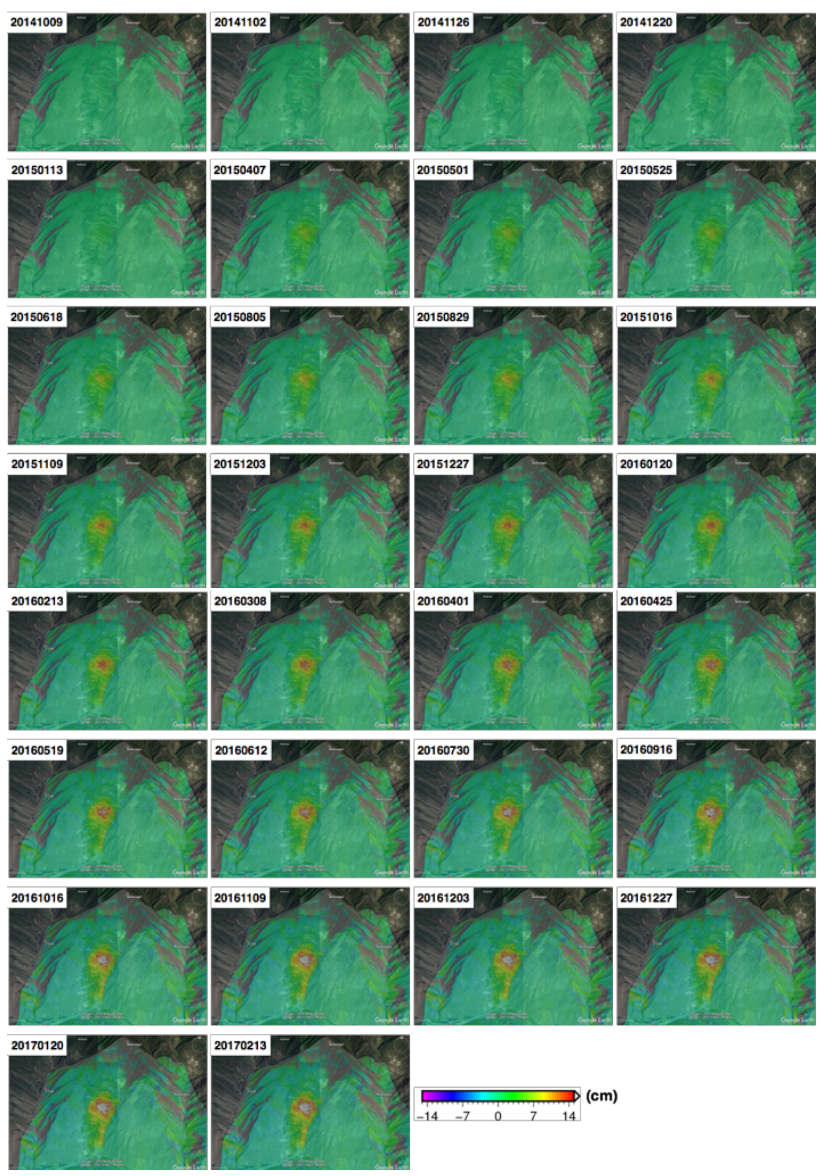

Figure 7. Time series evolutions of Xishancun Landslide acquired from Sentinel-1 datasets

In order to analyze the relationships between deformation evolution of borehole and rainfall, Figure 8 overlaps the data of daily rainfall measured by rain gauges with Sentinel-1 monitoring time series on Borehole 2, 5, 6, 7, 9 and 10. It can be seen from the image that the rainfall of Xishancun Landslide region is large from June to October every year, which is basically consistent with local weather forecast.

Borehole surface time series displacements showed to be gentle and approximately linear trend in dry seasons, however became fluctuating and accelerating in rainy seasons. The deformation acceleration trend of most boreholes influenced by seasonal rainfall has an obvious hysteresis effect.

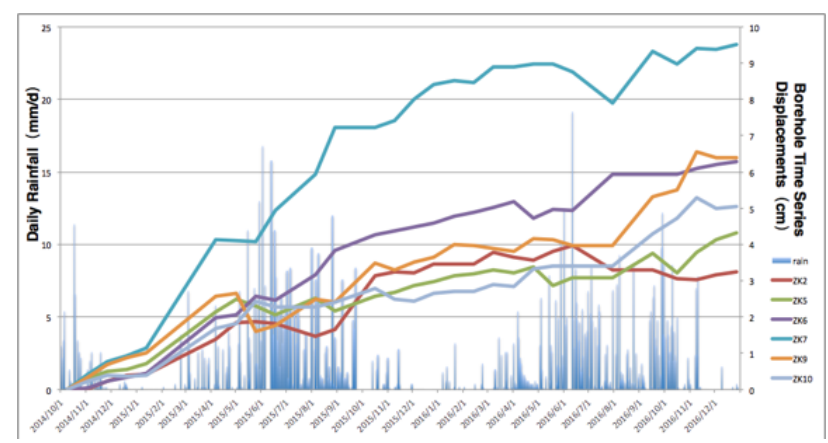

Figure 8 . Borehole time series displacements against the daily rainfall measurements

\section{CONCLUSIONS}

To fully investigate and validate the great potential of Sentinel-1 on landslide monitoring in complex terrain mountainous areas, and integrate the radar datasets from Sentinel-1 and TerraSAR-X, this work realized the landslide surface deformation acquisition with multi scales, short time intervals, and long time series, which also verify the great advantage of multi-platform spaceborne radar remote sensing on landslide monitoring. Combined with in situ measurements, landslide spatial temporal evolutions are further cultivated.

This work concludes that spaceborne radar remote sensing applications could demonstrate great potentials to monitor landslide deformations, identify the spatio-temporal characteristics and recognize landslide kinematics.

\section{ACKNOWLEDGEMENTS}

This work was supported by the 973 National Basic Research Program (No. 2013CB733204), National Key R\&D Program of China (No. 2018YFB0505300), Opening fund of State Key Laboratory of Geohazard Prevention and Geoenvironment Protection (Chengdu University of Technology) (SKLGP2019K022) and supported by State Key Laboratory of Geodesy and Earth's Dynamics (Institute of Geodesy and Geophysics, CAS) (SKLGED2019-5-1-E). The TerraSAR-X Stripmap datasets are copyrighted by DLR/Infoterra GmbH. The Sentinel-1 datasets are copyrighted by ESA. Some geocoded maps were generated by the General Mapping Tools (GMT).

\section{REFERENCES}

Barra, A.; Monserrat, O.; Crosetto, M., et al., 2017. In Sentinel-1 data analysis for landslide detection and mapping: First experiences in italy and spain, Workshop on World Landslide Forum; Springer: pp 201-208. 
Czikhardt, R.; Papco, J.; Bakon, M., et al., 2017. Ground stability monitoring of undermined and landslide prone areas by means of sentinel-1 multi-temporal insar, case study from slovakia. Geosciences, 7, 87.

Dai, K.; Li, Z.; Tomás, R., et al., 2016. Monitoring activity at the daguangbao mega-landslide (china) using sentinel-1 tops time series interferometry. Remote Sensing of Environment, $186,501-513$.

Fiaschi, S.; Mantovani, M.; Frigerio, S., et al., 2017. Testing the potential of sentinel-1a tops interferometry for the detection and monitoring of landslides at local scale (veneto region, italy). Environmental Earth Sciences, 76, 492.

Huang, R.; Li, W.L., 2009. Analysis of the geo-hazards triggered by the 12 May 2008 Wenchuan Earthquake, China. Bull. Eng. Geol. Environ., 68, 363-371.

Intrieri, E.; Raspini, F.; Fumagalli, A., et al., 2018. The maoxian landslide as seen from space: Detecting precursors of failure with sentinel-1 data. Landslides, 15, 123-133.

Kyriou, A.; Nikolakopoulos, K., 2018. Assessing the suitability of sentinel-1 data for landslide mapping. European Journal of Remote Sensing, 51, 402-411.

Li, Z.; Fielding, E.J.; Cross, P., 2009. Integration of insar time-series analysis and water-vapor correction for mapping postseismic motion after the 2003 bam (iran) earthquake. IEEE Transactions on Geoscience and Remote Sensing, 47, $3220-3230$.

Liu, C.; Li, W.; Wu, H.; Lu, P.; Sang, K.; Sun, W.; Chen, W.; Hong, Y.; Li, R., 2013. Susceptibility evaluation and mapping of China's landslides based on multi-source data. Nat. Hazards, $69,1477-1495$.

Liu C, Shao X, Wu H, et al., 2018. Giant landslide displacement analysis using a point cloud set conflict technique: a case in Xishancun landslide, Sichuan, China. International Journal of Remote Sensing: 1-20.

Liu X, Zhao C, Zhang Q, et al., 2018. Multi-Temporal Loess Landslide Inventory Mapping with $\mathrm{C}$-, X-and L-Band SAR Datasets-A Case Study of Heifangtai Loess Landslides, China. Remote Sensing, 10(11): 1756.

Qu, T.; Lu, P.; Liu, C., et al., 2016. Hybrid-sar technique: Joint analysis using phase-based and amplitude-based methods for the xishancun giant landslide monitoring. Remote Sensing, 8,874 .

Torres, R.; Snoeij, P.; Geudtner, D., et al., 2012. Gmes sentinel-1 mission. Remote Sensing of Environment, 120, 924.

Xu, Q.; Tang, M.; Xu, K., et al., 2008. Research on space-time evolution laws and early warning-prediction of landslides. Chinese Journal of Rock Mechanics and Engineering, 6, 005.

Ye, X.; Kaufmann, H.; Guo, X., 2004. Landslide monitoring in the Three Gorges area using D-InSAR and corner reflectors. Photogram. Eng. Remote Sens., 70, 1167-1172.
Yin, Y., 2009. Features of landslides triggered by the wenchuan earthquake. Journal of Engineering Geology, 1, 2938 .

Zhao, C.; Lu, Z.; Zhang, Q.; de La Fuente, J., 2012. Largearea landslide detection and monitoring with ALOS/PALSAR imagery data over Northern California and Southern Oregon, USA. Remote Sens. Environ., 124, 348-359. 